\title{
¿AUTISMO TECNOLÓGICO EN LOS ESTUDIANTES UNIVERSITARIOS? CASO PROVINCIA DE SANTA ELENA, ECUADOR
}

\author{
¿TECHNOLOGICAL AUTISM IN COLLEGE STUDENTS? THE CASE \\ OF SANTA ELENA PROVINCE, ECUADOR
}

\author{
Juan Ramos Peredo \\ juan.catedra@gmail.com \\ Universidad Tecnológica Equinoccial, Ecuador
}

\section{RESUMEN}

El presente estudio consistió en visualizar parámetros referenciales del autismo como patrón de conducta y aislamiento de los jóvenes peninsulares en sus actividades académicas y sociales, frente a una dependencia de los dispositivos móviles digitales, que tiene atrapada a una sociedad contemporánea. El estudio es de interés para todos los actores que se relacionan con ellos, sean sus padres, familiares, amigos, compañeros y docentes, justamente para tomar acciones que permitan su reinserción social o en algunos casos instruir a los estudiantes en el uso adecuado de los dispositivos móviles digitales. El artículo consistió en un trabajo de campo, encuestas y entrevistas a estudiantes, así como entrevistas a profesionales relacionados en las áreas de tecnología y psicología educativa. Entre los resultados obtenidos, se pudo conocer que un $74 \%$ de estudiantes encuestados denotan algún tipo de intranquilidad al no contar con un dispositivo móvil digital. Un $73 \%$ de alumnos, indica que por lo menos de una a dos veces usan el celular mientras dialogan personalmente. Entrevistas a estudiantes ilustran que algunos prefieren dialogar virtualmente que en persona con sus amigos o familiares. Estos resultados permiten inferir que existe dependencia y comportamientos de aislamiento social en los estudiantes puesto que a través del uso de los dispositivos móviles ven un medio de comunicación antes que hacerlo personalmente, así como también no podemos hablar de autismo tecnológico, puesto que no se ha comprobado en el ámbito científico ni médico.

Palabras clave: Autismo, tecnología, dispositivos móviles digitales, internet

\begin{abstract}
This study consisted in visualizing autism's reference parameters as pattern of behavior and isolation of young people in Santa Elena in their academic and social activities, compared to a reliance on digital mobile devices, which has trapped a contemporary society. The study is of interest to all stakeholders that are related to them are their parents, relatives, friends, classmates and teachers, in order to take actions that allow their reintegration into society or in some cases instructing students in the proper use of digital mobile devices. The article consisted of fieldwork, surveys and interviews to students, as well as interviews to professionals involved in the areas of technology and educational psychology. Among the results, it was known that $74 \%$ of students surveyed denote some kind of uneasiness at not having a digital mobile device. $73 \%$ of students, indicates that they use at least one or two times their cellphones while they converse personally. Interviews to students illustrate that some prefer to talk virtually than in person with their friends or family. These results allow us to infer that there is dependence and social isolation behaviors in students since through the use of mobile devices they see a way of communication instead of doing it personally, and also we cannot speak of technological autism, since it has not been proven in scientific or medical field.
\end{abstract}

Keywords: Autism, technology, digital mobile devices, internet

Recibido: 25 de noviembre de 2016

Aprobado: 10 de marzo de 2017

Publicado: 30 de junio de 2017 


\section{INTRODUCCIÓN}

El uso de la tecnología web forma parte de nuestras actividades cotidianas, en particular el contar con dispositivos móviles o también conocidos como smartphones con acceso a internet permiten mantenernos comunicados sin barreras ni fronteras, la socialización que se ha creado a partir de la optimización de internet, entre ellas están las redes sociales que permiten a las personas interactuar en un ambiente virtual, son una de las principales herramientas de comunicación. El número de usuarios que tiene cada una, ha aumentado de una manera muy acelerada gracias a las diversas utilidades que se les pueden dar: desde compartir información, fotos y pensamientos con gente en el aspecto personal, hasta promocionar productos $u$ ofrecer servicio al cliente en lo empresarial.

En este mismo sentido caben algunas interrogantes: ¿Qué tan prioritario o necesario resulta usar actualmente los dispositivos móvil?, ¿Qué tan dependientes somos del internet?, ¿Existe la adicción al internet?, ¿Cuánto tiempo se destina para interactuar en las redes sociales virtuales? Son algunas de las preguntas que se plantean a diario y si a esto se suman los comentarios de expertos en internet $y$ profesionales en psicología y sociología, se hace referencia a un tema que despierta polémica por los puntos de vista a favor y en contra que se exponen a su alrededor con respecto a las ventajas y desventajas de su uso.

Uno de los aspectos negativos que es sujeto de análisis está dado por la controvertida adicción a internet. Balaguer (2008, p.4) sostiene: "Ha sido casi inevitable, en estos últimos años, enfrentarme desde mi condición de psicólogo e investigador al impacto de las nuevas tecnologías; a la pregunta acerca de la adicción a Internet, de cómo es; qué características tiene; quiénes son los más vulnerables a padecer esta adicción, quiénes no; perfiles posibles de los adictos entre otras cuestiones". Este mismo autor expone: "He investigado estos fenómenos desde hace varios años, el creciente uso de Internet me ha enfrentado a situaciones clínicas de supuestas adicciones, tanto a este servicio como a otras tecnologías. El concepto de adicto a Internet es un concepto controvertido, algunos profesionales piensan que es una denominación válida (Young, 1996), mientras otros afirman que no se puede hablar de adicción a Internet (Matute, 2003). No ha habido acuerdo con respecto a ello, pero tanto en el imaginario social como en la prensa en general, se da por sentado su existencia".

Seguramente se observada a muchas personas por la calle, en un transporte público, en centros comerciales, direccionados en una sola actividad que es la de escribir en un dispositivo móvil digital gesticulando sin exponer una sola palabra con persona alguna, es una especie de soledad dentro de una multitud, cada día más se esparce por todo el planeta. En caso de nuestro país, no es la excepción.

En algún lugar se observan a individuos que prefieren estar solos, desarrollan un apego por los objetos en lugar de las personas, tienen risas o gritos sin motivos, hablan poco o nada, presentan movimientos repetitivos como balancearse, parecen sordos $\mathrm{y} / \mathrm{o}$ son hipersensibles a ciertos sonidos. Son alguna de las características de los "autistas tecnológicos", justamente se visualizan en ciertos sujetos que usan frecuentemente dispositivos móviles digitales.

El objetivo de este trabajo es mostrar el proceder relacionado con el uso que le dan a los dispositivos móviles digitales, como caso particular planteado a través de una gran interrogante: ¿Autismo tecnológico en los estudiantes universitarios de la Península de Santa Elena?

\section{METODOLOGÍA}

\section{Aplicación de técnicas de investigación}

Entre las técnicas de investigación que se utilizaron para la recolección de la información tenemos:

Investigación bibliográfica.

Observación directa.

Entrevistas y encuestas a alumnos y profesionales áreas de psicología e informática de universidades de la Península de Santa Elena.

Se aplicó un muestreo no probabilístico, seleccionando $300 \quad$ estudiantes aleatoriamente de diferentes niveles $y$ carreras de la Universidad Península de Santa Elena (UPSE) y Universidad Tecnológica Equinoccial (UTE) de una población de 1500 alumnos quienes fluctúan 
entre las edades de 18 y 20 años, de ambas entidades educativas.

\section{Desarrollo del concepto de autismo}

El término Autismo fue ideado por Bleuler en 1911, refiriéndolo originariamente a un trastorno básico de la esquizofrenia, que consistía en la limitación de las relaciones con las personas y con el mundo casi de manera extrema. De ahí las palabras Autismo y autista, que provienen del término griego autos que significa "sí mismo".

\section{Acerca de sintomatología del Autismo}

Lorna Wing (1988), describe doce dimensiones del desarrollo que consideraba siempre alteradas en los trastornos del espectro autista:

a) Relaciones sociales.

- Trastorno de la relación social.

- Trastorno de la referencia conjunta (acción, atención y preocupación conjunta).

- Trastorno intersubjetivo y mentalista.

b) Área de lenguaje y comunicación:

- Trastorno de las funciones comunicativas.

- Trastorno del lenguaje expresivo.

- Trastorno del lenguaje receptivo.

c) Área de flexibilidad mental y comportamental (conductual):

- Trastorno de la anticipación.

- Trastorno de la flexibilidad.

- Trastorno del sentido de la actividad propia.

d) Área de ficción e imaginación (simbólica):

- Trastorno de la ficción.

- Trastorno de la imitación.

- Trastorno de la suspensión.

\section{Desarrollo personal: ambiente conductual}

Kimble, Hirt, Díaz, Hosch, Lucker, y Zárate (2002, p. 40) exponen, un ambiente conductual es un hecho que se produce en un momento y lugar determinados, en el cual participan las personas y objetos del ambiente con un programa, funciones y roles a realizar. En un principio podemos escoger la situación donde nos encontraremos, pero una vez en ella la situación ejerce un influjo propio.

Ante lo expuesto en líneas precedentes, se infiere que el individuo está expuesto a recibir influencias del entorno, en este sentido es pertinente considerar que en ocasiones una de las razones además de la necesidad que tenemos por mantenernos informados a través de los dispositivos móviles digitales, es porque se considera que debemos estar al día en el uso de los mismos.

\section{Estar "Siempre encendidos" genera dependencia y estrés}

Según Gimeno (2014), algunas investigaciones científicas realizadas en los últimos años han demostrado que estamos inmersos de lleno en la era de la "celular dependencia", fenómeno que consiste en una especie de adicción a los dispositivos móviles, y especialmente provocado por la necesidad de la interconexión con el trabajo y con otras personas vía aplicaciones específicas como las redes sociales y las aplicaciones de mensajería instantánea. Y mientras más se incrementa el uso de los teléfonos inteligentes, mayor es la necesidad de estar siempre conectados, inclusive, en detrimento de la productividad laboral y de las relaciones interpersonales. Este fenómeno nos lleva a chequear los correos electrónicos del trabajo a toda hora, genera un cuadro de ansiedad extrema cuando vamos de vacaciones y no hay señal wi-fi o la locura que crea el hecho de no tener más batería en nuestro dispositivo. Este fenómeno es conocido en la actualidad como estar "siempre encendido", que es en definitiva, un grado importante de estrés.

\section{Soledad y tecnología}

Según Santrock (2006, p. 520), en la sociedad contemporánea, la tecnología constituye uno de los factores que puede generar soledad. A pesar de que la invención del teléfono hace más de un siglo parece haber contribuido a la disminución del aislamiento social que afecta a muchos individuos y familias, los psicólogos han determinado la existencia de un vínculo entre el consumo de televisión y los niveles de soledad. Esta relación no equivale a una relación causa y efecto, pero parece probable que la televisión pueda dar lugar a una situación de desconexión social.

Además, esta desconexión puede ser producida por el uso de internet, ya que muchos individuos se aíslan cuando se encuentran frente a sus ordenadores conectados a la red. En una investigación en la que se estudió a 169 individuos durante los primeros años en los que tuvieron acceso a internet (Kraut y otros, 1998), se concluyó que el uso elevado de internet se relaciona con una disminución en la comunicación de los sujetos con otros miembros de su familia $y$ con un aumento de las situaciones de 
depresión y soledad. Al mismo tiempo, no obstante, algunos individuos utilizan internet para establecer vínculos nuevos y estrechos (Clay, 2000).

\section{El autismo tecnológico: Phubbing}

Según Farfán (2013), en algún momento hemos vivido u observado que estando en una reunión, fiesta, restaurant, junta laboral y hasta en nuestras casas nos ha tocado compartir con alguien que no suelta su celular, nos sentimos incómodos frente a esta situación. No siempre estamos en la posibilidad de exponer algún comentario a la persona, y hasta cierto punto es lo que nos puede causar gran molestia.

El individuo que vive la imposibilidad de dejar de revisar los mensajes, correos electrónicos, conversar por intermedio de las redes sociales virtuales, se le conoce como phubbing, una mezcla de las palabras inglesas phone (teléfono) y snubbing (desairar), que define al acto de ignorar a alguien por mirar el teléfono.

\section{RESULTADOS Y DISCUSIÓN}

El presente estudio busca conocer la existencia de "Autismo Tecnológico" en los estudiantes universitarios de la Península de Santa Elena, como efecto del uso dependiente de dispositivos digitales.

Entre los principales resultados tenemos que el $75 \%$ de los estudiantes tienen internet permanente en sus celulares y un $25 \%$ no cuentan con ello, este resultado permite conocer que los universitarios tienen un alto acceso web.

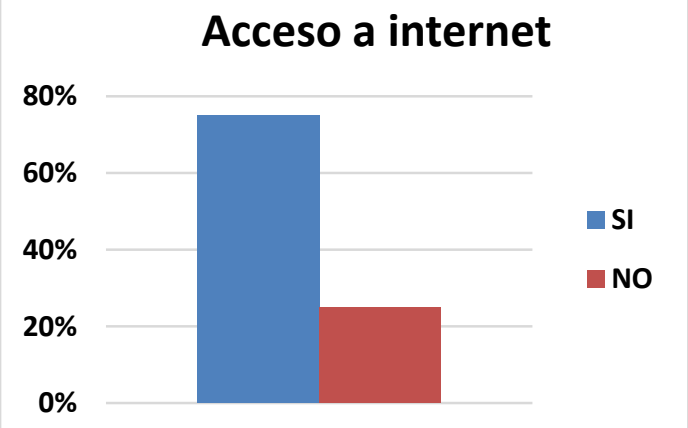

Figura 1. Porcentaje estudiantes que cuentan con internet en su celulares. Fuente: Encuesta a estudiantes de las universidades UPSE y UTE (2014)

En lo que tiene que ver con la utilización de los celulares para interactuar en las redes sociales, tenemos que el $87 \%$ de los alumnos interactúan con amigos y familiares, mientras que un $13 \%$ se dedica a conocer y relacionarse con nuevas personas. Estos resultados permiten visualizar una alta relación con personas allegadas a los estudiantes cuyo medio de comunicación están dados por los dispositivos móviles.

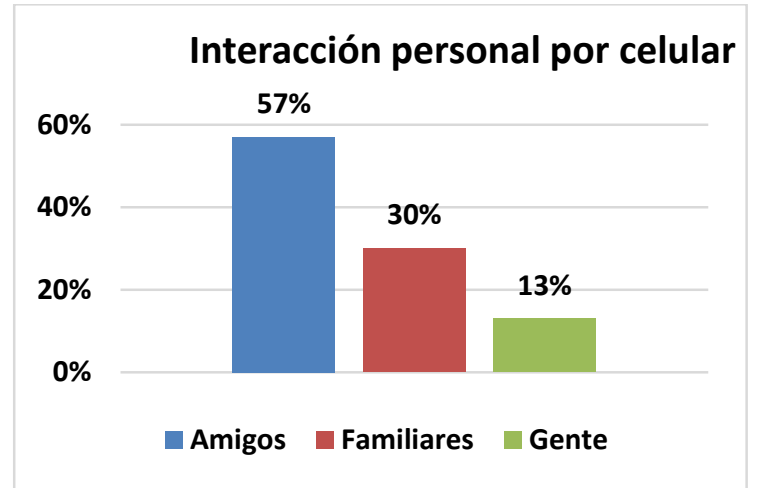

Figura 2. Interacción personal a través del celular. Fuente: Encuesta a estudiantes de las universidades UPSE y UTE (2014)

El siguiente resultado permite conocer la frecuencia de uso y al mismo tiempo se complementa con el resultado mostrado previamente puesto que el $82 \%$ de los encuestados se conectan siempre a internet correspondiendo a 248 de 300 encuestados a diferencia del $1 \%$ que nunca se conecta a internet siendo 3 de 300 encuestados.

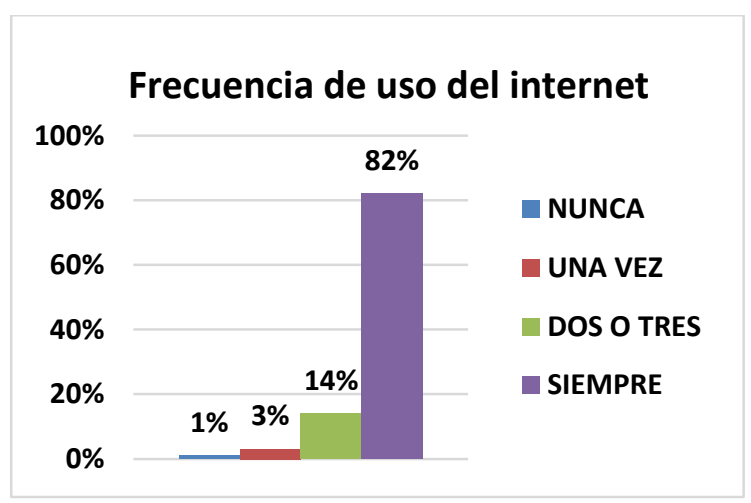

Figura 3. Nivel de frecuencia uso de internet en dispositivo móvil. Fuente: Encuesta a estudiantes de las universidades UPSE y UTE (2014)

Los estudiantes al no contar con su celular han expresado posturas que denotan desesperación, incomunicación e intranquilidad, esto es un total de $74 \%$. Este resultado representa un nivel considerable de dependencia y afectación en su persona ante la falta del dispositivo móvil. 


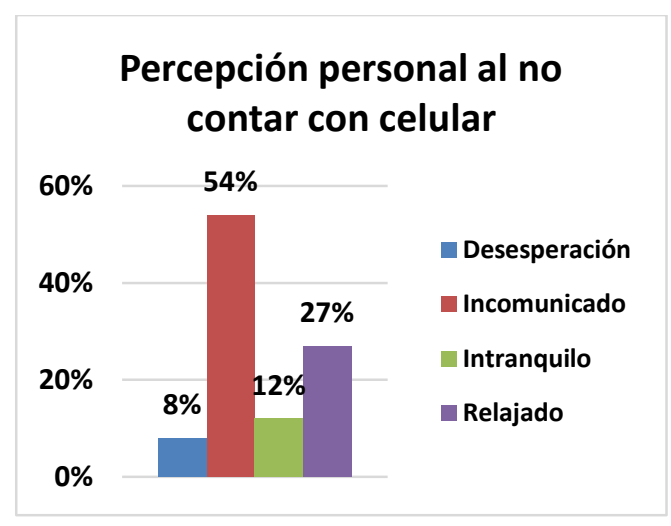

Figura 4. Percepción personal al no contar con celular. Fuente: Encuesta a estudiantes de las universidades UPSE y UTE (2014)

En lo que tiene que ver con las frecuencias de diálogos o conversaciones que los encuestados lo realizan a través de las redes sociales con otras personas, están dadas en el $57 \%$ y $32 \%$ lo hacen a diario y ocasionalmente vía online, a diferencia del $11 \%$ que no lo hacen. Estos resultados representan una preferencia bien marcada en cuanto al diálogo o contacto virtual que prefieren realizar los universitarios.

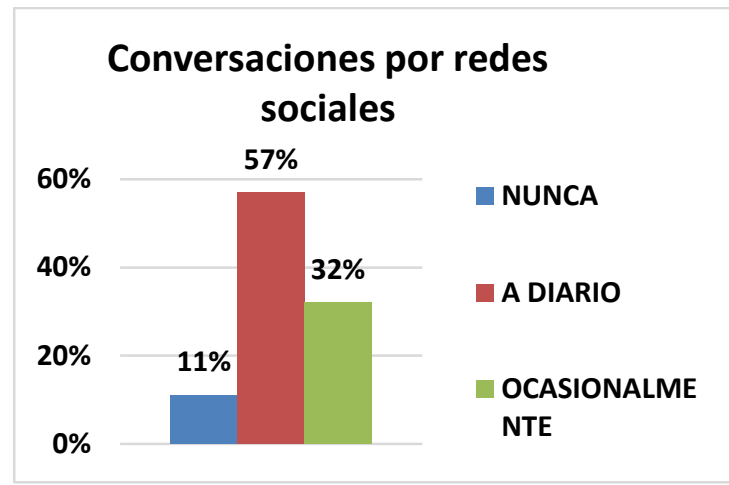

Figura 5. Nivel de conversación por redes sociales. Fuente: Encuesta a estudiantes de las universidades UPSE y UTE (2014)

Para conocer el número de veces que los encuestados usan el celular mientras dialogan personalmente, se conoció que un 50 y $23 \%$ de los alumnos Peninsulares utilizan por lo menos de una a dos veces respectivamente su dispositivo móvil. En esta pregunta resalta un término que apareció en el año 2007 junto a los teléfonos móviles inteligentes que se refieren al uso del mismo en presencia de otras personas, puede definirse como el acto de una persona al ignorar su entorno por concentrarse en su tecnología móvil ya sea un teléfono inteligente, tableta, laptop u otro dispositivo inteligente.

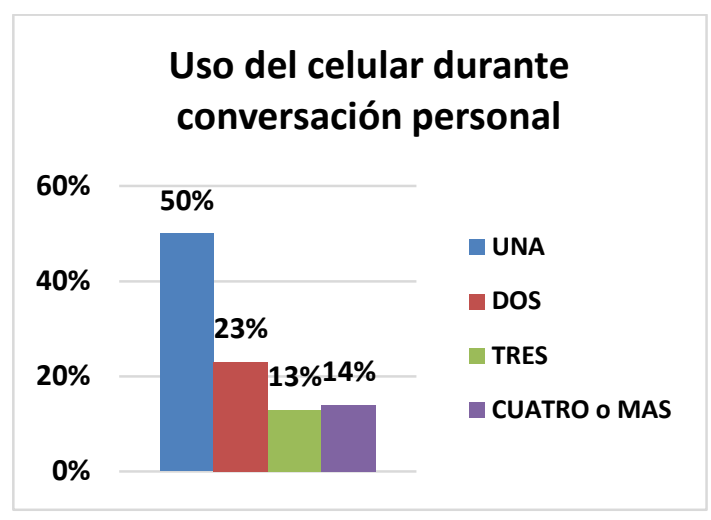

Figura 6. Número distracción (Phubbing) al dialogar personalmente. Fuente: Encuesta a estudiantes de las universidades UPSE y UTE (2014)

Las entrevistas y observaciones de campo, a los jóvenes universitarios de la UPSE y UTE, muestran, los estudiantes han olvidado la función por la cual fue creado el teléfono móvil, ya que pasó de ser un medio del cual se creía que su uso esencial, era acortar distancias y facilitar los momentos de emergencia, para pasar a ser una parte esencial en su vida cotidiana, que no solo le brinda comunicación, verbal y escrita con otras personas, si no también le brinda entretenimiento, esto se ha apoderado de sus vidas haciendo que actualmente se encuentre tanto ventajas como desventajas, las cuales van apareciendo según el uso que se esté haciendo.

Es importante que los usuarios de celulares, específicamente los jóvenes quienes son objeto de esta investigación, estén conscientes del uso que debe dársele al teléfono celular, ya que de lo contrario, se verán inmersos en una dependencia que le traerá problemas no solo psicológicos sino también físicos, esta situación es algo que podría controlarse para que no se torne perjudicial para la sociedad actual, la cual siente la necesidad del uso de la tecnología y estar al día en cuanto a las innovaciones.

Se entrevistaron a psicólogos de la UPSE y UTE, tal es el caso de Carlota Ordoñez Villao y Tanya Suárez Romero, respectivamente, quienes fueron consultadas sobre temas relacionados con la adicción, comportamientos, afectación social y familiar generados por el uso de dispositivos móviles. En cuanto al resultado de la entrevista a la psicóloga Carlota Ordoñez, considera que si existe adicción de los jóvenes a los 
dispositivos móviles, quienes están viviendo en la era digital 3.0 encaminándose a un autismo digital, expresado por una escasa comunicación humana. Así mismo expone que el cerebro se expande o tiene su plasticidad cuando existe el hecho de pensar y ponerse los problemas de situaciones 0 conflictos para resolverlos frente a la situación de otros seres humanos en contexto social.

El segundo problema que se confronta en esta situación es que el ser humano se olvida del ser humano y se proyecta frente a esta herramienta tecnológica. Y finalmente el ser humano se olvida de sus emociones y sensaciones, es decir no comparte con nadie, por lo tanto eso limita el crecimiento personal.

Por otra parte, ante la interrogante en cuanto a la existencia de semejanzas y comportamientos que podrían evidenciarse en una persona autista y otra con dependencia ante dispositivos móviles, infiere que el término autismo indica una escasez de la comunicación verbal es un problema neurológico identificado en el sistema nervioso de una neurona, en la red del ADN se especifica que hay una clasificación que la determina como tal.

Otras características están dadas por catarsis movimientos rituales, rutinas, hábitos constantes que no permite la llegada de otro ser humano para tener una relación social que su estructura y personalidad están totalmente desestructurados estamos hablando de una patología, pero cuando hablamos de autismo digital es un ser humano común y corriente pero que lastimosamente se encierra en sí en su computadora o dispositivo digital y no permite un espacio de comunicación, esta solo sometido a la realidad en el contexto de la computadora y el. Por eso le asemejan el término porque no tiene contacto social no hay una esfera de comunicación.

Continuando con la entrevista, la psicóloga Tanya Suárez Romero, manifestó que no ha tratado casos relacionados con la dependencia de dispositivos móviles, pero si ha observado a jóvenes que le dedican alto tiempo al uso de estos, inclusive cuando transitan por las calles, no observan por donde caminan.

Desde su punto de vista considera que si existe adicción al uso de los dispositivos móviles, así como la adicción a las sustancias químicas, adicción al alcohol, cigarrillo, a las compras, y el juego, el hecho de que te guste o estés muy "metido" en el dispositivo móvil, es una adicción.

En lo relacionado al término "autismo tecnológico", expresó que no lo ha escuchado como parte de las categorías de enfermedades mentales.

Frente a las semejanzas y comportamientos autistas y la dependencia de dispositivos móviles, la psicóloga Suárez sostiene que el autismo es un problema de comunicación, es un problema que puede tener una base orgánica o una base genética, la persona autista tiene problemas para comunicarse incluso hasta con su propia mamá; le afecta en su vida social, tiene su mundo, no hace contacto visual.

Existen personas autistas que inclusive tienen inteligencia muy alta pero en determinados factores, por ejemplo ha trabajado con gente autista, que son genios matemáticos y musicales. Se puede comparar únicamente en que la persona autista está ensimismada en su mundo, pero sólo eso, es gente que tiene problemas de lenguaje, no pueden estructurar una oración, tienen un problema conocido como ecolalia, repiten y repiten palabras pero no ha visto personas con esa dependencia tecnológica que tenga eso, son personas obsesionadas en cómo están colocadas las cosas que no le puedes mover algo porque se alteran fácilmente. La semejanza que le puede hallar es solo eso, que la persona esta ensimismada en su mundo y presenta problemas de pronto para comunicarse con los demás.

Este término "dependencia" si lo acepta, puesto que se lo está catalogando más como una "Dependencia". Afecta todo el entorno social y familiar del estudiante porque no se relaciona normalmente, no genera una relación del contacto humano, la misma se va perdiendo. La dependencia se refiere a aquellos jóvenes que pasan todo el día con el celular o computador, no interactúan con sus familiares y amigos normalmente, afecta la funcionalidad, cuando hay dependencia afecta a la vida de la persona.

Bajo el mismo concepto, se entrevistó al magister en sistema informáticos, Pepe Lascano, quien también es Ingeniero informático, con 22 años de experiencia y labores en el ámbito informático y se desempeña como docente e instructor de 
Ofimática en la Universidad Tecnológica Equinoccial.

El magister Lascano sostiene que la conducta de los estudiantes universitarios respecto al uso de los dispositivos móviles es acorde con el tiempo en que vivimos, la globalización, la tecnología y las comunicaciones nos obligan a que todos estemos a la par, se puede decir que casi todos los universitarios por no decir todos en realidad, deben tener un dispositivo móvil personal y la manera de comportarse es decir su conducta en una situación determinada o en general, y si lo utiliza para bien o para mal, pero esto en realidad va a ser muy difícil dar un juicio de valor, porque tendríamos que analizarlos en ese aspecto individualmente, por más que se le pueda preguntar al estudiante, por ejemplo, cuándo utilizan el celular con una conducta positiva o negativa, se atrevería a decir que todos lo hacen de manera positiva.

Desde el punto de vista pedagógico y en su opinión personal, no comparte que los estudiantes utilicen dispositivos móviles en el aula, puesto que no existe una cultura en la utilización estrictamente académica por parte del estudiantado, hoy en día existen tantas aplicaciones, redes sociales, chats que distraen y tientan al estudiante a su uso, si eso fuera controlado, cosa muy difícil, sería un éxito.

En lo relacionado con rendimiento de los estudiantes al usar dispositivos móviles, expresa que es muy bajo, si el estudiante se dedica a chatear, cosa que ha sucedido, dichos alumnos han tenido que salir de clase para que acaben de "conversar, chatear, etc.", y entren a la siguiente hora clase, lo cual ocasiona atrasos en las materias.

Así mismo considera que si existe adicción al uso de los celulares, totalmente es muy evidente no solo los estudiantes también los profesores, y otras personas.

Para el magister Lascano, el término "Autismo Tecnológico" o algún otro término relacionado no le es familiar, mientras que si ha evidenciado ciertos comportamientos en los estudiantes, cuando no cuenta con los Smartphone, entre ellos: nerviosismo, intranquilidad y desesperación.

Finalmente considera que la dependencia del dispositivo móvil afecta el entorno social y familiar del estudiante, tanto así que cae en una falta de respeto, para las personas que son sujeto de desatención en el momento que se encuentran con otra persona, a tal punto que va aumentado la carencia de valores que hoy en día se ha perdido considerablemente.

En las secciones precedentes se visualizaron conceptos y criterios que describen el autismo como una enfermedad que es atendida clínicamente basado en manifestaciones conductuales individuales, donde una de sus afectaciones puede reflejarse en ambientes sociales.

Por otra parte, un ambiente conductual induce a un individuo a satisfacer una necesidad por mantenerse comunicado e informado a través de un dispositivo móvil, generando un uso habitual e interacciones humanas básicamente virtuales. En este mismo sentido, la conducta inductiva frente al uso de los dispositivos móviles en público, genera otros comportamientos que denotan en el individuo un aislamiento en algunos casos momentáneos o temporales llegando al desaire e ignorando a alguien por observar el celular.

\section{CONCLUSIONES}

Esta investigación expuso criterios de diversos psicoanalistas y profesionales que estudian el comportamiento humano a nivel individual, grupal y social; así como también todas aquellas variables 0 factores endógenos, exógenos y tecnológicos que de alguna manera generan influencias en el accionar de las personas. Particularmente en lo relacionado con el uso de los dispositivos móviles digitales, no obstante los criterios que se vierten ante ello reflejan semejanzas, diferencias y posturas a favor o en contra del uso de los mismos.

Se dio a conocer que en la persona autista se ven afectadas fundamentalmente doce dimensiones enmarcadas en cuatro grupos: dimensión social, comunicación y lenguaje, conductual y simbólica. Entre las características más representativas tenemos aquellas que afectan la comunicación con otras personas, el aislamiento con otros individuos de su entorno, dependencia simbólica denotando conductas estereotipadas con objetos. Las características antes descritas, obedecen a dictámenes estrictamente clínicos, basados en manifestaciones conductuales. 
El ambiente conductual es un hecho que se ve afectado por la interacción de personas y cosas, por lo que el individuo está expuesto a recibir influencias del entorno, ante esto, el uso de los dispositivos móviles digitales además de mantenernos informados, es porque se considera que debemos estar a la moda o al día en el uso de los mismos. Particularmente estos criterios que pudieron visualizar en las respuestas que concedieron los estudiantes de las universidades UPSE y UTE.

La aplicación de encuestas a los estudiantes universitarios visualizó que un $75 \%$ cuentan con internet en sus celulares, relacionado con una permanencia en la web de un $82 \%$. Entre otros resultados tenemos posturas o actitudes que manifiestan dependencia ante la falta del dispositivo móvil digital, como es el caso de desesperación, incomunicación e intranquilidad.

Otro aspecto relevante en la encuesta a los estudiantes, está dada por la afectación estereotipada con un objeto, como es el uso del dispositivo móvil digital cuando conversan personalmente con otro par o persona, es decir, mientras dialogan tienden a ignorar su entorno y prestan atención a su dispositivo digital, esta acción actualmente recibe el nombre de "phubbing".

Psicólogos y profesionales en informática relacionados con las entidades universitarias, en su experiencia concluyen en la existencia de una dependencia por parte de sus estudiantes en cuanto al uso y en algunos casos; por otra parte, no se puede hablar de un "autismo tecnológico" puesto que esta terminología no ha sido evaluada científicamente.

Finalmente, se concluye que existe dependencia del uso de dispositivos móviles digitales, los mismos que responden al tiempo tecnológico en que vivimos, a un mundo globalizado y comunicado sin fronteras. Ante aquello, no podemos hablar de "autismo tecnológico" en los estudiantes peninsulares de las universidades UPSE y UTE, ya que para ello deberíamos contar con sintomatologías que describan conductas comprobadas y aceptadas a nivel científico y médico por los organismos mundiales competentes, a pesar de ciertos comportamientos que guardan alguna semejanza conductual con personas autistas.

\section{REFERENCIAS BIBLIOGRÁFICAS}

1. Alonso, J., Blanco, S., y Blanco, S. Tecnologías de la información y de la comunicación. México: Editorial Alfaomega. 2005.

2. Arrebillaga, M. Autismo y trastornos del lenguaje. Argentina: Editorial Brujas. 2010. Recuperado de http://site.ebrary.com/lib/utesp/docDetail. action?doc $\mid \mathrm{D}=10390150$

3. Balaguer, R. ¿Adicción a Internet o adicción a la existencia? Revista Digital Universitaria, 2008. 9 (8), 1-12. Recuperado de http://www.revista.unam.mx/vol.9/num8/a rt55/int55.htm

4. Balaguer, R. La adicción a Internet en Adicción. 2001. Montevideo: Cuadernos de la Coordinadora de Psicólogos del Uruguay. Recuperado de http://www.revista.unam.mx/vol.9/num8/a rt55/int55.htm

5. Cabrera, D. Generalidades sobre el autismo. Revista Colombiana de Psiquiatría, 2009. 36. Recuperado de http://www.scielo.org.co/scielo.php?pid= S003474502007000500016\&script=sci_arttext

6. Carroll, David. Psicología del lenguaje, (4 ed) España: Editorial Paraninfo. 2008.

7. Cleffi, V. Trastorno del Espectro Autista (TEA). 2015. Recuperado de http://www.ms.gba.gov.ar/sitios/pediatria/ trastorno-del-espectro-autista-tea/

8. Cuxart, F. Evolución conceptual del término "autismo": una perspectiva histórica. Revista de Historia de la Psicología. 1998. volumen 19 (2-3), 369$388 . \quad$ Recuperado de http://www.revistahistoriapsicologia.es/ap p/download/5971828211/36.\%2BCUXAR T\%2BI\%2BFINA.pdf\%3Ft\%3D13921995 $50+\& \mathrm{~cd}=5 \& \mathrm{hl}=\mathrm{es}-419 \& \mathrm{ct}=\mathrm{clnk} \& \mathrm{gl}=\mathrm{ec}$

9. De Acedo, M.L. Psicología: Mente y Conducta, (2da. Ed.). España: Editorial Desclee De Brouwer. 1998

10. Etchegoyen, $\mathrm{H}$. Los fundamentos de la técnica psicoanalítica, (3era. Ed.). Buenos Aires: Editorial Amorrortu. 2002.

11.Farfán, M. T. El autismo tecnológico. 2013. Phubbing. Recuperado de http://www.atomoeducativo.org/phubbing/

12.Gimeno, L. Estar "Siempre encendidos" genera dependencia y estrés. 2014. Radio Canadá Internacional. Recuperado de

http://www.rcinet.ca/es/2014/08/28/estarsiempre-encendidos-generadependencia-y-estres/ 
13. Harmut, R. El autismo, (2da. Ed.) Madrid: Editorial Acento. 1997.

14.Kimble, C., Hirt, E., Dìaz, R., Hosch, H., Lucker, W., y Zàrate, M. Psicología Social de las Américas, (1era. Ed.) México: Editorial Pearson. 2002.

15.Santrock, J. Psicología del desarrollo: EI ciclo Vital, (10ma. Ed.). Madrid, España: Editorial Mc Graw Hill. 2006.

16.Tallis, J. Los trastornos del espectro autista: aportes convergentes. Argentina: Editorial Miño y Dávila. 2012 Recuperado de
http://site.ebrary.com/lib/utesp/docDetail. action?docID $=10831666 \&$ p00 =autismo

17.Vera, J. Ética, tecnología y sociedad, Chile: Ediciones Universidad Central de Chile. 2001.

18.Wing, L. El autismo en niños y adultos: Una guía para la familia, (1ra. Ed.). Barcelona: Editorial Paidos lbérica. 1988.

19. Woolfolk, A. Desarrollo Humano, (11ma. Ed.). México: Editorial Prentice Hall. (2010). 